Jérôme Jacquin

\title{
S'opposer à autrui en situation de co-présence : la multimodalité de la désignation contre-argumentative
}

\begin{abstract}
Avertissement
Le contenu de ce site relève de la législation française sur la propriété intellectuelle et est la propriété exclusive de l'éditeur.

Les œuvres figurant sur ce site peuvent être consultées et reproduites sur un support papier ou numérique sous réserve qu'elles soient strictement réservées à un usage soit personnel, soit scientifique ou pédagogique excluant toute exploitation commerciale. La reproduction devra obligatoirement mentionner l'éditeur, le nom de la revue, l'auteur et la référence du document.

Toute autre reproduction est interdite sauf accord préalable de l'éditeur, en dehors des cas prévus par la législation en vigueur en France.
\end{abstract}

\section{revues.org}

Revues.org est un portail de revues en sciences humaines et sociales développé par le Cléo, Centre pour l'édition électronique ouverte (CNRS, EHESS, UP, UAPV).

Référence électronique

Jérôme Jacquin, "S'opposer à autrui en situation de co-présence : la multimodalité de la désignation contreargumentative », Semen [En ligne], 39 | 2015, mis en ligne le 19 novembre 2015, consulté le 28 janvier 2016. URL: http://semen.revues.org/10467

Éditeur : Presses universitaires de Franche-Comté

http://semen.revues.org

http://www.revues.org

Document accessible en ligne sur :

http://semen.revues.org/10467

Document généré automatiquement le 28 janvier 2016.

(c) Presses universitaires de Franche-Comté 


\section{S'opposer à autrui en situation de co-présence : la multimodalité de la désignation contre-argumentative}

\section{Introduction}

La présente contribution se propose d'aborder la manière, fondamentalement pluri-sémiotique ou « multimodale », dont le discours vis-à-vis duquel le contre-discours se positionne est désigné1. En d'autres termes, on s'intéresse aux différents processus et ressources mobilisés par un opposant pour désigner, pointer, faire référence à la source de son opposition. On se concentre en outre sur un type de contre-discours particulier : la contre-argumentation, telle qu'elle se manifeste dans des situations où tous les participants sont co-présents, aussi bien temporellement que spatialement.

Cette question est intéressante dans la mesure où l'opposition constitue une pierre de touche de la plupart des théories dialectiques de l'argumentation (Van Eemeren \& Grootendorst 2004, Plantin 2005), dès lors considérée comme une manière particulière de gérer un désaccord (à différencier de l'agression physique, de l'insulte, de l'humour ou du simple déni). En d'autres termes, c'est bien du contre-discours qu'émerge la confrontation qui donne son sens à l'argumentation. En 2011, je m'étais exclusivement concentré sur la dimension linguistique de la désignation, en liant l'opposition argumentative aux différentes ressources relevant de la délégation énonciative, et plus précisément de la représentation du discours autre et de la polyphonie (Jacquin 2011, voir également 2014). J'aimerais ici revenir sur la question en considérant l'apport d'une approche multimodale pour l'appréhension du phénomène.

Adopter une perspective multimodale revient à prendre acte du caractère incarné, incorporé, de tout phénomène d'énonciation et à considérer ainsi le tressage des différentes conduites sémiotiques dans la construction méthodique du sens en contexte (Sidnell \& Stivers 2005, Streeck, Goodwin \& LeBaron 2011, Deppermann 2013). S'agissant d'analyser des phénomènes argumentatifs en situation de co-présence, on s'intéresse dès lors à la manière dont les unités linguistiques et les procédés discursifs s'articulent à la direction du regard, aux pointages gestuels ou encore à la disposition des corps dans l'espace.

Après une brève présentation des données soumises à l'analyse (2.), je résumerai l'approche discursive de l'argumentation - et, partant, de la contre-argumentation - sur laquelle la présente contribution repose (3.). On définira ensuite les rapports de places tels qu'ils s'inscrivent dans la contre-argumentation, en général, et dans les données considérées, en particulier (4.). On aura alors une meilleure idée de ce en quoi une « désignation contreargumentative » consiste et on pourra alors entrer dans le vif du sujet, à savoir le caractère fondamentalement multimodal - et dynamique - de ces phénomènes (5.). Après la présentation du cadre d'analyse (5.1.), je développerai l'analyse d'un cas particulier où le tressage des différentes ressources désignatives endosse différents enjeux communicationnels relatifs aux rapports de places en présence (5.2. et 5.3.).

\section{Corpus et données}

Les données considérées sont tirées d'un corpus vidéo-enregistré de huit interactions publiques - de types débat ou conférence-discussion -organisées par des associations d'étudiants sur différentes thématiques politiques (l'écologie, le financement des études supérieures, l'influence des médias sur la politique, etc.). S'étant déroulées sur le campus de l'Université de Lausanne, ces pratiques sont fondamentalement ancrées dans la démocratie semi-directe suisse qui appelle les citoyens à voter plusieurs fois par an sur des objets divers, qu'il s'agisse d'un contexte d'initiative (une proposition citoyenne visant à modifier 
la constitution) ou de référendum (une proposition citoyenne visant à bloquer une loi adoptée par le Parlement). Ce système politique particulier, doublé d'un fédéralisme privilégiant les particularités cantonales, contribue à entériner un certain rapport à l'espace public, par la multiplication d'événements ponctuels visant à faire se rencontrer citoyens et politiciens dans des cadres minimalement médiatisés, comme ceux analysés ici ${ }^{2}$. S'ils s'apparentent aux « débats citoyens » analysés par Burger (2009), les événements documentés ici témoignent davantage d'une politique de terrain et de proximité, où seul un animateur vient médier la co-présence spatiale et temporelle de l'ensemble des participants, public compris (pour plus de détails sur l'élaboration de ce corpus, voir Jacquin 2014). Ces données rendront ainsi particulièrement visible la tension que le pointage contre-argumentatif fait intervenir entre l'intersubjectivité qui caractérise le rapport entre les deux locuteurs en situation de désaccord et l'espace public en tant qu'il positionne le public-citoyen comme un tiers-témoin.

\section{Une approche discursive de l'argumentation}

La présente contribution s'inscrit dans un courant de réflexion spécifique, le modèle dialogal de l'argumentation, développé par Plantin (1996, 2005) et enrichi par Doury (1997, 2003). Fondamentalement linguistique et descriptive, cette approche s'inspire de la tradition rhétorique classique (en particulier Hermogène) et contemporaine (Bitzer 1968) et met au cœur de son dispositif théorique, méthodologique et analytique l'idée -qui ne va pas de soi - que l'argumentation constitue une manière verbale de gérer une divergence d'opinions. Une situation argumentative émerge ainsi de la « cristallisation d'un désaccord » (Traverso 2005), c'est-à-dire de l'élaboration méthodique et de la consolidation progressive de positions antagonistes autour d'une question commune.

Plus précisément, l'argumentation sera définie ici comme

un mode spécifique de traitement verbal du désaccord, qui consiste en la construction de positions solides, c'est-à-dire étayées par un travail textuel de justification et situées par un travail interactionnel et dialogique de positionnement. (Jacquin \& Micheli 2012 : 600)

La place manque pour expliquer en détail en quoi le modèle dialogal de l'argumentation ainsi que cette définition qui s'en inspire se distinguent d'autres traditions, des plus linguistiques aux plus discursives, des plus spécifiantes aux plus généralisantes (voir sur ce point Jacquin \& Micheli 2012, Jacquin 2014). On retiendra toutefois que l'approche invite à adopter une perspective interdisciplinaire au sein des sciences du langage, en situant le phénomène à l'articulation du dire (problématique de l'enchaînement textuel et de la prise en charge énonciative des énoncés) et du faire ensemble (problématique de la coordination et de la négociation interactionnelles des tours de parole).

En ce sens, l'approche permet de questionner la manière dont le désaccord argumenté passe par la construction d'une position comme venant se confronter à une autre position, précédemment exprimée. Deux dimensions de ce processus sont particulièrement pertinentes pour la présente contribution. Il s'agit d'une part des enjeux identitaires - ou rapports de places - relatifs non seulement à l'argumentation en général, mais aussi au genre de rencontre considéré (le débat public dans le cas présent). Il s'agit d'autre part des enjeux sémiotiques de la désignation, c'està-dire de la diversité des ressources référentielles permettant au contre-discours de pointer le discours contesté. Ce sont bien ces enjeux sémiotiques qui nous intéressent ici, mais leur étroite articulation aux catégorisations identitaires justifie qu'on introduise brièvement ces dernières.

\section{Contre-argumentation et rapports de places}

Les différentes approches dialectiques de l'argumentation - qu'il s'agisse du modèle dialogal cité supra ou du modèle pragma-dialectique d'Amsterdam (pour une synthèse, voir Van Eemeren \& Grootendorst 2004) - insistent particulièrement sur le fait qu'argumenter instancie certains rôles complémentaires, c'est-à-dire inscrit le locuteur dans un rapport de places particulier $^{3}$ :

[d]ans sa forme prototypique, la situation argumentative apparaît comme une situation d'interaction entre discours du Proposant et contre-discours de l'Opposant, médiatisée par un 
discours Tiers, donc une situation de « trilogue », qui s'incarne de façon exemplaire dans l'échange public contradictoire. (Plantin $2005: 64$ )

La situation argumentative prototypique s'actualise de fait différemment selon le genre de discours considéré. Ainsi, il est tout à fait possible - et prévu - que la situation argumentative soit produite de manière monologale. C'est le cas du monologue intérieur, où un même locuteur fait se confronter dialogiquement le discours et le contre-discours et ainsi alterne entre les places de Proposant et d'Opposant. La particularité des situations analysées ici (voir supra, 2.) réside dans le fait qu'elles engagent un développement à la fois interactionnel et séquentiel de la situation argumentative prototypique, dans le sens où les places sont prises et négociées par des agents différents, au fil de l'interaction. C'est "l'échange public contradictoire » auquel Plantin fait référence et qui engage un «cadre de participation » particulier (Goffman 1987). Ainsi, dans les débats publics, la place du Tiers est prise non seulement par un animateur-médiateur chargé de transposer dans l'interaction in praesentia la Question faisant débat dans l'espace public, mais aussi par le public, assistant à la confrontation et régulièrement encouragé à questionner directement les intervenants invités. Et ce sont bien entendu ces invités - politiciens ou experts plus ou moins partisans - qui se distribuent séquentiellement les places d'Opposant et de Proposant, en prenant alternativement en charge le discours et le contre-discours. Notons encore qu'il n'est pas rare qu'un membre du public quitte momentanément sa place de Tiers-témoin pour prendre part à la confrontation en venant s'opposer à un invité. Si ces mécanismes sont bien connus (voir déjà Greatbatch 1992, Hutchby 1996), il n'en va pas de même de la complexité sémiotique qui caractérise les désaccords argumentés en situation de co-présence et, plus précisément, de la manière multimodale dont le contre-discours désigne, pointe vers le discours auquel il se confronte. Compte tenu du cadre de participation qu'on vient de décrire, l'examen des phénomènes de désignation contre-argumentative dans les débats publics nous invitera également à considérer la tension, stratégiquement exploitable par l'Opposant, qui lie intersubjectivité (s'adresser à autrui), délocution (parler d'autrui) et publicisation (parler devant autrui).

\section{La multimodalité de la désignation contre-argumentative}

Je commence par brièvement présenter le cadre d'analyse retenu pour l'appréhension du phénomène (5.1.), en distinguant les types de ressources concernées (5.1.1.) et en précisant leurs modes de combinaison (5.1.2.). On se concentrera alors sur un cas particulier de complémentarité entre ressources verbales et ressources mimo-gestuelles (5.2.), avant d'en synthétiser les effets communicationnels (5.3.).

\subsection{Cadre d'analyse}

\subsubsection{Les différents types de ressources : verbalité et mimo-gestualité}

Les ressources verbales (RV)

Par définition, toute contre-argumentation suppose un dialogisme, même minimal, c'est-à-dire un ancrage énonciatif d'autrui et de son discours dans sa propre parole. Formulé différemment, analyser la contre-argumentation exige de porter une attention particulière aux ressources permettant de prendre en compte un discours, sans pour autant le prendre en charge (Coltier, Dendale \& De Brabanter 2009). Ce dialogisme contre-argumentatif repose sur un très large éventail de ressources énonciatives, allant de la représentation du discours autre (AuthierRevuz 2004) aux phénomènes de polyphonie (Bres et al. 2005). ${ }^{4}$.

Avec la première, il s'agit pour le locuteur de faire lire ou de faire entendre à nouveau un discours - les propos - et avec lui le point de vue - l'opinion - qu'il véhicule. Les différentes techniques disponibles sont bien connues et vont des plus mimétiques et détachées (le discours direct) aux plus synthétiques et intégrées (le discours narrativisé), en passant par des cas mixtes ou intermédiaires comme le discours indirect ou la connotation/modalisation autonymique (voir également Rosier 1999). Comme on le verra, la capacité de la représentation du discours autre (désormais RDA) de fonctionner comme désignation contre-argumentative repose en grande partie sur l'appareil attributif mobilisé, et en premier lieu sur l'usage qui est fait des 
ressources dites référentielles (Charolles 2002) : déictiques personnels (tu, nous, vous) ou anaphoriques impersonnels (illelle, ils/elles) ${ }^{5}$, formes dites «d'adresse » (monsieur Untel) ou encore syntagmes nominaux (la personne à ma droite, la présidente du parti socialiste). En l'absence de ressources référentielles, c'est le degré de mimétisme et le potentiel écho avec un discours précédemment tenu qui peut permettre à la RDA d'en assurer la désignation. Ce mécanisme indirect de désignation nous renvoie au second cas.

Celui-ci est plus diffus et donc plus complexe. La polyphonie ne passe en effet pas par l'enchâssement d'un discours dans un autre, mais instancie directement, simultanément au discours produit, un point de vue autre qui se trouve en relation hiérarchique avec le point de vue principal, véhiculé par le discours. C'est particulièrement sensible avec certains connecteurs (mais, puisque), mais aussi avec la négation dite polémique (ce mur n'est pas blanc), qui permet de prendre en compte un point de vue (ce mur est blanc) tout en prenant en charge sa contestation (Ducrot 1972, Nølke 1992). La capacité de la polyphonie à fonctionner comme désignation contre-argumentative dépend dès lors fortement de la proximité temporelle et/ou spatiale des énoncés (par exemple lorsqu'un tour de parole en suit un autre dans un dialogue, ou que deux textes écrits sont disposés côte à côte). En d'autres termes, si le phénomène polyphonique est seul, c'est-à-dire sans accompagnement par un pointage du regard ou de la main / du doigt, son potentiel désignatif dépendra exclusivement de la présence d'un écho avec un énoncé précédent, par exemple dans la séquence typique de question-proposition-opposition qui caractérise l'activité de débattre (Jacquin 2014).

Les ressources mimo-gestuelles (RMG)

17 Les ressources mimo-gestuelles couvrent au moins deux grands types de phénomènes : la direction du regard et l'accomplissement de gestes (mimiques faciales comprises). Comme pour les ressources verbales, on brossera ici un bref panorama des RMG en tant qu'elles peuvent assurer une désignation.

18 Pour ce qui est du regard dans l'interaction verbale, le récent état de l'art proposé par Rossano (2013) fournit différents éléments pertinents pour notre propos. On notera tout d'abord que le regard est un phénomène éminemment culturel, ce qui implique, comme pour les ressources verbales, de se garder de généralisations abusives. On notera ensuite que les cultures occidentales utilisent le regard pour instancier l'allocutaire, mais que cette instanciation ne se fait toutefois pas sur le mode d'un regard « insistant» ou «soutenu », qui véhicule des informations supplémentaires (séduction ou agression, selon les cas). Ainsi, si la « home position » (Sacks \& Schegloff 2002) du regard est bien la personne à qui on s'adresse, on note de fréquents phénomènes de « décrochement » lors desquels le regard est disponible pour désigner et assurer une attention conjointe sur autre chose que l'allocutaire. Toutefois, regarder quelqu' un d'autre que l'allocutaire de manière appuyée, à un point séquentiel particulier ou en combinaison avec des ressources verbales particulières, fonctionnera comme un changement d'allocutaire, voire comme une sollicitation (voir par exemple Stivers \& Rossano 2010).

Pour ce qui est des gestes, la panoplie de valeurs est bien entendu très large. Toutefois, une catégorie de gestes renvoie précisément à ce qui nous occupe ici : les gestes de pointage et, plus précisément, les gestes d'ostension. Ceux-ci pointent littéralement sur des éléments du contexte d'énonciation et d'interaction (sur le pointage-en-interaction, voir par exemple Hindmarsh \& Heath 2000, Goodwin 2003, Kendon 2004 : 199-224). Quelle que soit son amplitude ou sa rapidité (Enfield, Kita \& de Ruiter 2007), cette référence déictique permet dès lors de concentrer précisément l'attention conjointe sur un objet et d'assurer la référence indépendamment ou parallèlement à ce qui se joue non seulement au niveau langagier mais aussi au niveau de la direction du regard.

20 Dans les deux cas, les spécialistes insistent sur l'importance du contexte séquentiel de production (Goodwin 2003, Kendon 2004, Rossano 2013). Et par là, il ne s'agit pas de considérer seulement l'environnement actionnel immédiat (l'action précédente, l'action suivante), mais aussi, de manière plus diffuse, la tâche - ou activité finalisée - à l'accomplissement duquel la ressource participe. Dans le cas présent, on considérera les ressources mimo-gestuelles dans les limites où elles participent à indexer et ancrer l'activité contre-argumentative en situation de co-présence. 


\subsubsection{La coordination des ressources : redondances et différences}

Pour chaque type de ressource, il s'agit donc de considérer non seulement son extension référentielle (le contour de ce qui est désigné par la ressource), mais aussi son positionnement séquentiel (le moment précis où elle est mobilisée). Extension référentielle et positionnement séquentiel constituent ainsi deux axes de variation, qui permettent d'envisager un éventail de possibilités d'articulation entre ressources verbales et mimo-gestuelles dans l'accomplissement d'une désignation contre-argumentative. Des cas de «pures » redondances sont certes envisageables, que ce soit sur l'axe séquentiel ou référentiel, mais il s'agit d'une possibilité logique et non empirique, qu'il s'agirait de fonder sur une analyse de l'orientation multimodale des locuteurs vers l'appréhension de ces phénomènes.

Ainsi, dans le cas suivant, où un intervenant (SANS) en interrompt très localement un autre (BONJ) pour s'opposer argumentativement à lui (lignes 7-8), SANS désigne non seulement BONJ du regard (image 1), mais il utilise également le pronom « vous » (ligne 7), avant de revenir à une position neutre en fin d'énoncé (image 2$)^{6}$ :

\section{Extrait 1 / ETU-EMP / 01:50'00"'}

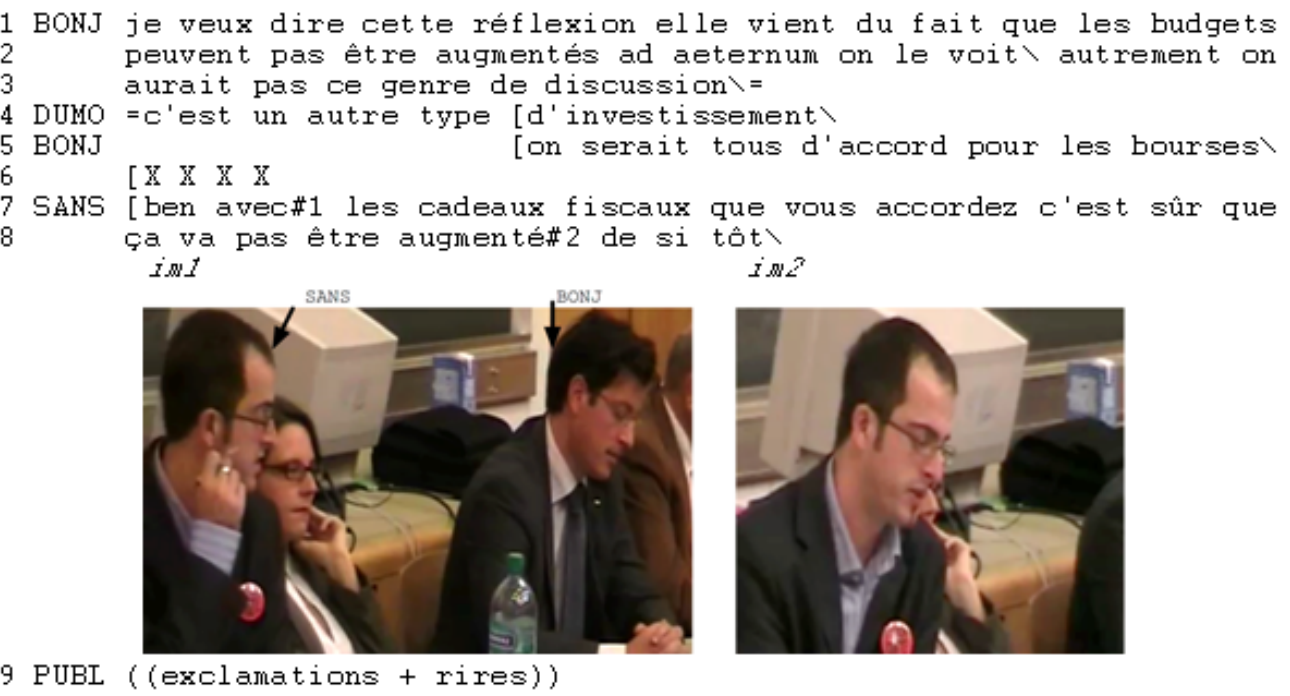

Même dans ce cas en apparence si simple, « vous » n'équivaut pas à «tu » en français, du fait que ce pronom personnel peut relever soit d'un « vous» de politesse (le locuteur désigne son adversaire uniquement), soit d'un « vous » pluriel multipliant les allocutaires ou, comme ici, incluant l'adversaire dans un groupe que le locuteur prend à parti (l'équivalent d'un « vous et ceux qui êtes avec vous »). Polysémique et, dans le cas présent, non restreint référentiellement par le sémantisme de l'énoncé (à distinguer de « vous êtes un menteur »), le pronom « vous » permet d'étendre l'extension référentielle de la désignation opérée par le regard, dynamique peut-être favorisée encore par le décrochement de ce même regard en fin de tour.

Les analyses qui suivent privilégieront les situations où les disjonctions référentielles et temporelles sont particulièrement sensibles, c'est-à-dire lorsque la désignation fait l'objet d'un travail multimodal lourd impliquant une coordination complexe entre ressources verbales et mimo-gestuelles.

\subsection{Analyse d'un cas}

25 Plus précisément, je vais me concentrer sur le cas de double disjonction le plus représenté - et de très loin - dans les données considérées : lorsque les RMG viennent restreindre $a$ posteriori l'extension référentielle initiale'. En d'autres termes, le locuteur entame sa contreargumentation par un cadrage désignatif flou ou du moins large, dans le sens où ni les RMG ni les RV ne viennent désigner au moins un participant en particulier. Dans un second temps toutefois, le locuteur use de la mimo-gestualité pour préciser l'extension référentielle initiale et ainsi ancrer le point de vue contre lequel il argumente. 


\subsection{1. «On a beau critiquer... »}

Cet extrait est tiré du même événement que le précédent. Alors que le thème de la rencontre est « quelles études aujourd'hui pour quels emplois demain ? », l'animateur a proposé aux dix intervenants invités d'entamer le débat par un tour de table consacré aux modèles de financement des études supérieures à privilégier. L'extrait est tiré de la dernière intervention du tour de table, celle de Dumoulin (DUMO), qui va localement s'opposer à une prise de position précédemment exprimée à l'encontre du processus de Bologne.

\section{Extrait 2 / ETU-EMP / 00:37'50"'}

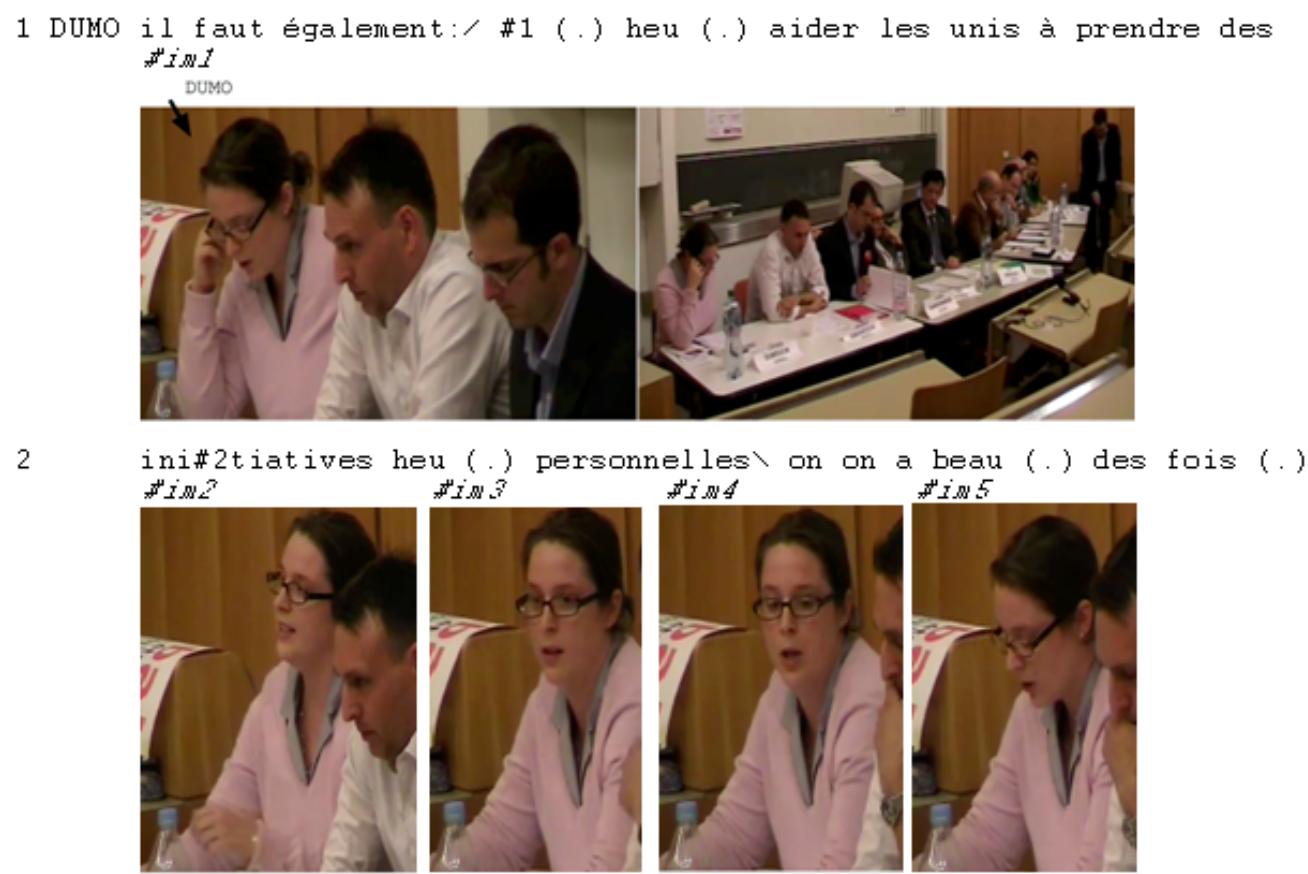

critiquer\#3 heu\#4 bologne/\#5 mais je trouve que c'est quand même un un très beau projet $/$ (...) qui a été pris' (...) l'initiative a été prise PAR les universités/(.) et au début il n'y a pas eu d'intervention des états du tout/

La locutrice commence par énoncer la conclusion (« il faut»), sous la forme d'un encouragement aux « initiatives personnelles» prises par les universités. Au moment de prononcer « initiatives », DUMO passe d'un regard baissé sur une feuille disposée devant elle (image 1) à un regard dirigé vers le public (image 2). DUMO poursuit ensuite non pas en citant directement l'exemple du processus de Bologne comme argument à l'appui, mais en marquant le caractère contre-argumentatif de son énoncé. L'énoncé de DUMO « on a beau des fois critiquer Bologne » relève d'un cas de RDA intéressant, car il autorise une très large extension référentielle. L'infinitif « critiquer » permet à la locutrice d'éviter toute mention de l'agent à l'origine du procès (à distinguer de « vous critiquez »); il représente le discours ciblé dans sa virtualité (Riegel, Pellat \& Rioul 2009 : 580), comme un discours à la fois énoncé et énonçable dans l'espace public. Cette indétermination de l'agent est en outre renforcée par le « on », qui « rejet[te] [le] référent dans l'anonymat» (voir note 5) et donc entretient le flou référentiel. Finalement, cette RDA constitue une synthèse des propos d'autrui (cas de discours dit «narrativisé»), qui évite ainsi l'idiosyncrasie d'un discours direct sur le mode de la citation (Rosier 1999).

L'indétermination de cette désignation est toutefois compensée, progressivement et dans un second temps, par un regard qui, bien que fugace et relativement flou du point de vue de son extension référentielle, instancie un ou des participant(s) positionné(s) sur la gauche de la locutrice (images 2, 3 et 4). En d'autres termes, le regard vient incarner et par conséquent préciser l'extension référentielle laissée indéterminée par le «on » et l'infinitif «critiquer», en « arrimant » le point de vue non pris en charge (être critique envers Bologne) à des positions précédemment exprimées dont la locutrice produit en quelque sorte un écho polyphonique. 


\subsection{2. "Prétendre qu'il suffit »}

Dans l'extrait suivant, les deux invités, Blanc (BLAN) et Nantermod (NANT), ont été conviés à débattre la question « Financer les études par des bourses ou par des prêts ? ». Les thèses défendues par les intervenants sont présentées comme antagonistes dès leur présentation par l'animateur : alors que le premier invité soutient une augmentation des moyens alloués aux bourses d'études, le second propose un système de prêts dans lequel l'étudiant rembourserait le prêt dont il a bénéficié au prorata de son revenu à la sortie de ses études. L'extrait est tiré de la fin de l'événement, lorsque s'achève la prise de parole du dixième membre du public à s'être exprimé (PUB10).

\section{Extrait 3 / PRE-BOU / 00:49'09"'}

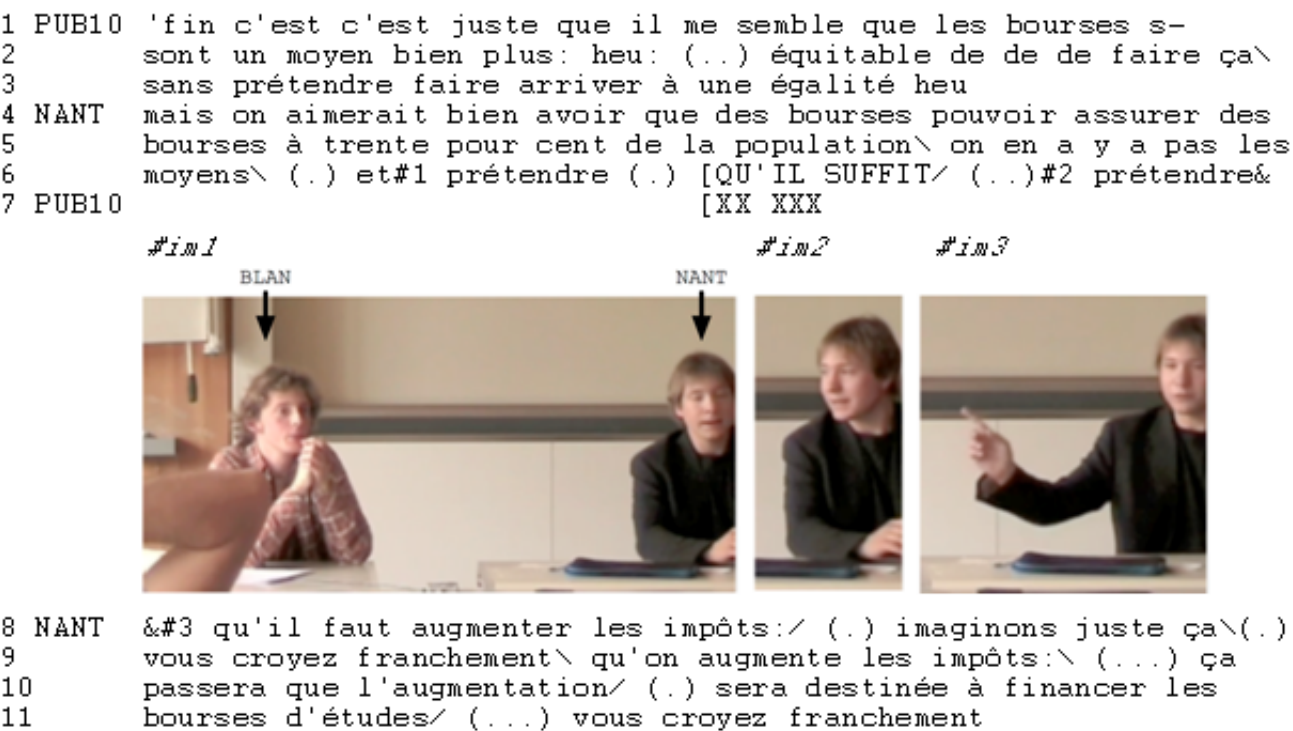

En 1-3, PUB10 présente les bourses d'études comme un moyen «plus équitable [que les prêts] » de parvenir à l'égalité sociale. NANT débute alors son tour avec un «mais » qui signale d'entrée de jeu la nature contre-argumentative de son tour vis-à-vis de la position exprimée par PUB10. NANT oppose ainsi à la possibilité d'une généralisation des bourses d'études un principe de réalisme financier («y a pas les moyens »).

La suite du tour est plus intéressante, dans la mesure où NANT va progressivement intégrer BLAN comme cible de son mouvement contre-argumentatif. NANT enchaîne en effet avec un usage particulièrement intéressant de la RDA et plus précisément du verbe «prétendre », énoncé deux fois («prétendre qu'il suffit, prétendre qu'il faut augmenter les impôts », lignes 6-8). On retrouve ici l'usage de l'infinitif qui, à la différence de «il prétend » ou «tu prétends », n'exprime pas le sujet de l'action, mais permet de présenter le discours représenté (« qu'il suffit, qu'il faut augmenter les impôts ») dans sa virtualité et dans sa disponibilité en tant que discours déjà en circulation. Cette extension référentielle large se combine avec le sémantisme du verbe «prétendre», qui ne se confond pas avec d'autres verbes de RDA de type « dire » ou « affirmer ». Le locuteur tente en fait un coup de force qui lui permet de signaler que lui-même autant que l'opinion commune (la « doxa ») se distancient des propos rapportés (voir sur ce point les analyses de Berrendonner 1981). Ce cas de RDA est donc clairement contre-argumentatif, orienté contre un discours en circulation, potentiellement endossable par n'importe qui.

Toutefois, considérer le positionnement séquentiel respectif des deux occurrences de « prétendre » ainsi que la mimo-gestualité qui les accompagne invite à revoir l'analyse, particulièrement en ce qui concerne la seconde occurrence. La première occurrence de «prétendre » est accompagnée d'un regard flottant qui renforce clairement le caractère désancré du propos contesté. En d'autres termes, l'extension référentielle reste large. La seconde occurrence est par contre précédée d'un changement de direction du regard (vers BLAN, image 2) et accompagnée d'un geste de pointage particulièrement saillant (image 3) venant se substituer au regard (qui retourne en situation initiale). En d'autres termes, le 
discours en circulation instancié par « prétendre » est en quelque sorte « arrimé » à BLAN et l'extension référentielle de la contre-argumentation se trouve réduite.

Ainsi, tout en continuant à s'opposer directement à PUB10, dont le statut d'allocutaire est maintenu par le regard, NANT montre qu'il ne perd pas de vue le contexte et le rapport de places dans lequel s'inscrit sa prise de parole : il s'agit certes de répondre à PUB10, mais aussi de poursuivre la confrontation avec son adversaire BLAN, face au public. Par ce «prétendre » et cet usage particulier de la mimo-gestualité, PUB10 et BLAN se trouvent dès lors réunis dans un ensemble auquel il est possible pour NANT d'opposer des arguments communs.

\subsection{3. “Cette façon de dire "ah..." »}

34 Le dernier extrait suit le précédent de quelques dizaines de secondes et constitue l'amorce de l'ultime prise de parole de BLAN.

\section{Extrait 4 / PRE-BOU / 00:50'53"'}

1 BLAN alors je trouve juste que c'est bizarre cette façon de dire ah\#1 तHIMI

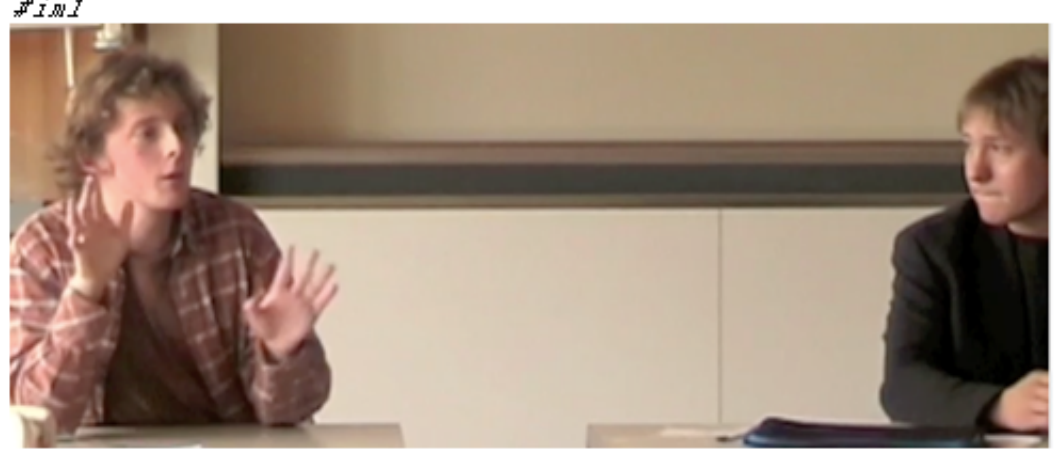
qu'y a à berne\#3 et les g- les gens veulent pas c'est une drôle

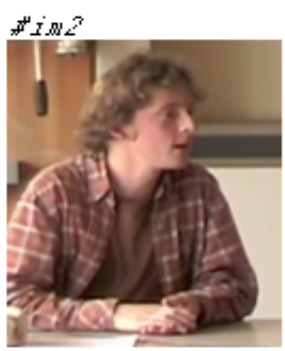
形工似

4 de façon de faire de la politique quand on fait de la politique 5 on a quand même l'idée pour changer les choses (..) on prend pas

Le procédé énonciatif est à la fois semblable et différent du précédent. Il est semblable de par l'utilisation d'un verbe d'attribution de parole à l'infinitif ( «dire », ligne 1), qui virtualise le discours représenté, le débusque sans pour autant l'attribuer. BLAN évite ainsi de limiter la responsabilité énonciative de l'énoncé à un locuteur précis. Le procédé est toutefois différent, car il ne repose pas sur le mode indirect de l'enchâssement syntaxique ( «dire que... »), mais sur le mode du discours direct (DD), de la citation, détachée, autonome (« ah augmenter les impôts de toute façon ils voudront pas... »). Dans le cas du DD, il est admis que le locuteur fait une «mention de message » (Authier-Revuz 2003 : 82), c'est-à-dire qu'il prête sa voix, et ce à proprement parler, à un autre que lui. Quel que soit le degré de fidélité « réelle » au discours d'origine, le DD propose ainsi un contrat de lecture de l'ordre du mimétisme : ce que je formule, c'est ce qu'un autre a prononcé. Par ce principe de littéralité, le DD parait restreindre le champ des possibles référentiels qu'autorisait l'infinitif, mais il n'y a toutefois aucune attribution explicite du DD à NANT. Finalement, une autre différence avec le cas précédent tient au choix du verbe introducteur. Alors que «prétendre » dispose d'une orientation argumentative spécifique, ce n'est pas le cas de « dire », dont la perspective est neutre. C'est par conséquent l'environnement sémantique du verbe, et plus précisément les formules attributives respectivement cataphorique « c'est bizarre » (ligne 1) et anaphorique «c'est une drôle de façon » (lignes 3-4), qui marque la distanciation et ainsi oriente argumentativement le discours représenté. 
Au plan mimo-gestuel, on constate que BLAN oriente progressivement son regard vers NANT (image 2), mais qu'il attend aussi la fin du discours représenté sur le mode direct pour le désigner véritablement (image 3). Cette désignation mimo-gestuelle accompagne la dislocation à droite du syntagme nominal « les élus qu'y a à berne », qui à la fois ancre référentiellement le « ils » précédent et enchaîne sur la dernière proposition « et les gens veulent pas ». BLAN maintient donc mimo-gestuellement la détachabilité de l'énoncé qu'il rapporte, avant de finir par le rattacher par le regard à son adversaire, qui évite alors toute réciprocité en baissant les yeux (image 3) alors qu'il regardait BLAN en début de tour (image 1).

\subsection{Synthèse du cas}

Comme on l'a dit, ce phénomène de disjonction, ou du moins de complémentarité, entre les contenus véhiculés respectivement par les ressources verbales et mimo-gestuelles est particulièrement fréquent dans les données considérées. Cela s'explique probablement par une loi d'économie : en situation de co-présence, les ressources mimo-gestuelles sont en quelque sorte disponibles pour ne pas être limitées à la redondance informationnelle et permettent ainsi aux locuteurs d'accomplir plus d'une chose à la fois.

Plus précisément, pour ce qui est du cas considéré, des ressources verbales particulières comme l'infinitif ou le « on » permettent au locuteur de laisser indéterminées, dans un premier temps, les personnes susceptibles d'adopter la position incriminée. Le locuteur garantit ainsi la lisibilité et la généralité des positions argumentées en les extrayant des contingences locales de l'énonciation. Dans un deuxième temps, il ré-arrime en quelque sorte le discours contesté à l'adversaire par un geste de pointage ou par la direction du regard, qui glisse les propos rapportés dans la bouche de l'adversaire. Le locuteur rétablit ainsi la contextualité, y compris corporelle, du débat en ré-ancrant les rapports de places en présence : le locuteur se montre corporellement, face au public, comme restant engagé dans une confrontation in praesentia avec un participant incarnant la position incriminée. Ce ré-arrimage, bien que saillant, est toutefois fugace : (i) s'agissant du regard, il s'agit d'éviter une réciprocité visuelle dont l'adversaire pourrait profiter pour prendre son tour en jouant sur l'effet de sollicitation ${ }^{8}$; (ii) s'agissant du regard comme du pointage gestuel, il s'agit de valoriser le caractère spectaculaire de la confrontation, qui fait dès lors du public le témoin d'une forme d'agression symbolique (Burger, Jacquin \& Micheli 2011). La désignation mimo-gestuelle permet ainsi de faire référence à l'adversaire tout en conservant la portée générale de l'argumentation, et ainsi d'associer autrui à une position contre laquelle on s'oppose.

\section{Conclusion}

La présente contribution visait à enrichir l'étude de la désignation par une prise en compte de l'apport de la mimo-gestualité à la construction du sens en contexte. Ce contexte, tout aussi déterminant que réflexivement construit par les ressources mobilisées (Duranti \& Goodwin 1992), c'était l'activité contre-argumentative d'une part, et le genre du débat public de l'autre. On s'est ainsi intéressé à un cas particulier de disjonction temporelle et référentielle entre les instructions données respectivement par les ressources verbales et mimo-gestuelles et on a considéré les effets de cette désignation contre-argumentative dans des situations argumentatives caractérisées par la co-présence des participants.

Sur cette base, plusieurs prolongements seraient envisageables. Il s'agirait déjà de proposer une analyse systématique et contrastive des cas restants. Quant aux phénomènes pouvant bénéficier d'une analyse multimodale, l'unité « on » est apparue à l'analyse comme un candidat particulièrement pertinent. 


\section{Conventions de transcription}

\begin{tabular}{ll} 
Conventions adaptées de ICOR (v. 2007, ICAR). \\
$/$ & $\begin{array}{l}\text { Intonation montante } \\
\text { Intonation descendante }\end{array}$ \\
$\vdots$ & Allongement phonique \\
\hline & Troncation \\
& $\begin{array}{l}\text { Pauses }(1 / 4,1 / 2,3 / 4 \text { de seconde) et silences } \\
\text { (n secondes) }\end{array}$ \\
XX XXX $(.).(\mathrm{n})$ & Segment inaudible (X= environ une syllabe) \\
MAIS & Emphase \\
{$[$ YY YYYY] } & Segment chevauché \\
$=$ & Enchaînement immédiat \\
$\&$ & Prolongation du tour après interruption \\
$($ (rit) $)$ & Commentaire \\
$\# 1$ \#im1 & Image 1
\end{tabular}

Résumé : L'argumentation peut être définie comme une manière verbale, parmi d'autres, de gérer un désaccord. Elle repose dès lors sur l'idée d'un contact entre, d'une part, une position soutenue dans un discours et, de l'autre, sa contestation dans un contre-discours. La présente contribution se propose d'aborder la manière, fondamentalement pluri-sémiotique ou "multimodale », dont ce contact est géré et ce désaccord exprimé dans des situations de co-présence, c'est-à-dire lorsque les locuteurs engagés dans la confrontation argumentée sont réunis dans un même lieu en un même moment. Ces situations rendent à la fois disponible et pertinent un large éventail de ressources - représentation du discours autre, polyphonie, direction du regard, pointages gestuels - et permettent de considérer la complexité sémiotique qui caractérise la manière dont le discours vis-à-vis duquel le contre-discours se positionne est désigné. Les analyses se fondent sur un corpus vidéo-enregistré de débats publics organisés par des associations d'étudiants sur différentes thématiques politiques. Ces données rendent particulièrement visible la tension que la désignation contre-argumentative fait intervenir entre intersubjectivité (rapport qui s'établit entre les deux locuteurs en situation de désaccord) et publicisation (rapport qui s'établit avec le public-citoyen positionné comme tiers-témoin).

Mots-clés : argumentation ; multimodalité ; représentation du discours autre ; polyphonie ; mimogestualité

Abstract : Argumentation can be considered as a specific way of managing disagreements and conflicts. Such a definition stresses the importance of the contact between a position that is defended in a discourse and its contestation in a counter discourse. This paper analyzes the multimodal way this contact is managed and the disagreement expressed in situations where all the participants are temporally and spatially co-present. These situations rely on the use of different types of resources such as reported speech, polyphony, gaze direction and pointing gestures. They are therefore of particular interest for analyzing the multimodal strategies that the opponent uses to designate the origin/source of his/her opposition, namely the confronted position and the person who expressed it. The paper is based on data taken from a video-recorded corpus of eight public debates, which have been organized from 2007 to 2009 at the University of Lausanne by student associations on topics ranging from ecology to the funding of universities. The data show how counter argumentative designations relate both to intersubjectivity (i.e., the relationship between the participants who experience the disagreement) and publicization (i.e., the relationship with the public, which is established as the third-party).

Keywords : argumentation ; multimodality ; reported speech ; polyphony ; gaze and gestures

\section{Bibliographie}

AUTHIER-REVUZ, J., (2003), Le Fait autonymique : Langage, langue, discours. Quelques repères. In Authier-Revuz, J., Doury, M., \& Reboul-Touré S., (éds.), Parler des mots : le fait autonymique en discours. Paris : Sorbonne Nouvelle, 67-96.

- (2004), La représentation du discours autre : un champ multiplement hétérogène. In Munoz J.M.L., Marnette S., \& Rosier L., (éds.), Le discours rapporté dans tous ses états. Paris : L’Harmattan, 35-53. 
BERRENDONNER, A., (1981), Éléments de pragmatique linguistique. Paris : Minuit.

BITZER, L.F., (1968), « The Rhetorical Situation », Philosophy and Rhetoric, 1(1), 1-14.

BRES, J., HAILlET, P.P., MELLET, S., NØLKE, H., \& ROSIER, L., (éds.), (2005), Dialogisme et polyphonie : approches linguistiques. Bruxelles : De Boeck.

BURGER, M., (2009), « Le cadrage de la communication dans les médias : apports d'une analyse linguistique », Communication / Université Laval, 27(2), 18-50.

BURGER, M., JACQUIN, J., \& MICHELI, R., (éds.), (2011), La parole politique en confrontation dans les médias. Bruxelles : De Boeck.

CHAROLLES, M., (2002), La référence et les expressions référentielles en français. Paris : Ophrys.

COLTIER, D., DENDALE, P., \& DE BRABANTER, P., (2009), « La notion de prise en charge : mise en perspective », Langue française, 162(2), 3-27.

DEPPERMANN, A., (éd.), (2013), Conversation Analytic Studies of Multimodal Interaction, Journal of Pragmatics, 46(1).

DOURY, M., (1997), Le débat immobile. L'argumentation dans le débat médiatique sur les parasciences. Paris : Kimé.

- (2003), «L'évaluation des arguments dans les discours ordinaires. Le cas de l'accusation d'amalgame », Langage et société, 105, 9-37.

DUCROT, O., (1972), Dire et ne pas dire. Paris : Hermann.

DURANTI, A., \& GOODWIN, C., (éds.), (1992), Rethinking Context : Language as an Interactive Phenomenon. Cambridge : CUP.

ENFIELD, N.J., KITA, S., \& DE RUITER, J.P., (2007), « Primary and secondary pragmatic functions of pointing gestures », Journal of Pragmatics, 39(10), 1722-1741.

FLAHAULT, F., (1978), La parole intermédiaire. Paris : Seuil.

GOFFMANN, E., (1987), Façons de parler. Paris : Minuit.

GOODWIN, C., (2003), « Pointing as Situated Practice». In KITA, S., (éds.), Pointing. Where language, culture, and cognition meet. Mahwah, NJ : Lawrence Erlbaum Associates, 217-241.

GREATBATCH, D., (1992), « On the management of disagreement between news interviewees ». In DREW, P., \& HERITAGE, J., (éds.), Talk at Work: Interaction in institutional settings. Cambridge : CUP, 268-301.

HINDMARSH, J., \& HEATH, C., (2000), « Embodied reference: A study of deixis in workplace interaction », Journal of Pragmatics, 32(12), 1855-1878.

HUTCHBY, I., (1996), Confrontation Talk : arguments, asymmetries, and power on talk radio. Mahwah, N.J. : Lawrence Erlbaum Associates.

JACQUIN, J., (2011), «Localiser, décrire et faire voir le fait argumentatif : le modèle dialogal de l'argumentation au défi d'un corpus complexe », A contrario, 16, 88-109.

— (2014), Débattre. L'argumentation et l'identité au coeur d'une pratique verbale. Bruxelles : De Boeck.

JACQUIN, J., \& MICHELI, R., (2012), «Entre texte et interaction : propositions méthodologiques pour une approche discursive de l'argumentation en sciences du langage ». In NEVEU, F., MUNI TOKE, V., BLUMENTHAL, P., KLINGLER, T., LIGAS, P., PRÉVOST, S., \& TESTON-BONNARD, S., (éds.), Actes du 3ème Congrès Mondial de Linguistique Française, Lyon : EDP Sciences, 599-611.

KENDON, A., (2004), Gesture : Visible Action as Utterance. Cambridge : CUP.

NØLKE, H., (1992), « Ne... pas : négation descriptive ou polémique? Contraintes formelles sur son interprétation », Langue française, 94, 48-67.

PLANTIN, C., (1996), « Le trilogue argumentatif. Présentation de modèle, analyse de cas », Langue française, 112, 9-30.

— (2005), L'argumentation : histoire, théories et perspectives. Paris : PUF.

RIEGEL, M., PELLAT, J.-M., \& RIOUL, R., (2009), Grammaire méthodique du français. Paris : PUF. ROSIER, L., (1999), Le discours rapporté : histoire, théories, pratiques. Bruxelles : De Boeck.

ROSSANO, F., (2013). «Gaze in conversation ». In SIDNELL, J., \& STIVERS, T., (éds.), The Handbook of Conversation Analysis. Chichester : Wiley-Blackwell, 308-329.

SACKS, H., \& SCHEGLOFF, E.A., (2002), « Home position », Gesture, 2(2), 133-146. 
SIDNELL, J., \& STIVERS, T., (éds.), (2005), Multimodal Interaction, Semiotica, 156.

STREECK, J., GOODWIN, C., \& LEBARON, C., (éds.), (2011), Embodied Interaction: Language and Body in the Material World. Cambridge : CUP.

TRAVERSO, V., (2005), «Cristallisation des désaccords et mise en place de négociations dans l'interaction: des variations situationnelles ». In GROSJEAN, M., \& MONDADA, L., (éds.), La négociation au travail. Lyon : PUL, 43-69.

VAN EEMEREN, F.H., \& GROOTENDORST, R., (2004), A Systematic Theory of Argumentation : the pragma-dialectical approach. Cambridge : CUP.

Z'GRAGGEN, H., \& LINDER, W., (2004), Professionalisierung der Parlaments im internationalen Vergleich. Studie im Auftrag der Parlamentsdienste der Schweizerischen Bundesversammlung. Berne : Institut für Politikwissenschaft.

\section{Notes}

1 La présente publication bénéficie du soutien du Fonds National Suisse de la recherche (FNS), par le biais d'une bourse Early Postdoc. Mobility octroyée à l'auteur pour un séjour de recherche de 12 mois à la Victoria University of Wellington, Nouvelle-Zélande ( ${ }^{\circ}$ P2LAP1_155110). L'auteur tient à remercier la relectrice anonyme pour son évaluation et ses suggestions. Il prend toutefois l'entière responsabilité de toute maladresse qui subsisterait.

2 Encore que le terme de «politicien » soit trompeur s'agissant de la Suisse, qui se caractérise en effet par la très faible professionnalisation de sa sphère politique (Z'graggen \& Linder 2004).

3 Pour des raisons expliquées ailleurs (voir Jacquin 2014 : 294-296), je privilégie la notion de « place » à celle de « rôle », utilisée par Plantin dans la citation qui suit. La notion de « rapports de places » est quant à elle reprise de Flahault (1978).

4 Les manières de définir et de distinguer les notions de dialogisme, de représentation du discours autre et de polyphonie varient parfois fortement d'une approche à l'autre, voire d'un auteur à l'autre. Les distinctions n'étant pas fondamentales ici, je m'en tiens au consensus possible que j'ai adopté précédemment (Jacquin 2014).

5 On portera également une attention toute particulière au pronom on, dont Riegel, Pellat \& Rioul nous disent qu'il « vérifie pleinement sa définition dans les mots croisés : "un vague sujet". Sa valeur de base est, en effet, celle d'un pronom indéfini renvoyant à une personne ou à un ensemble de personnes d'extension variable, que le locuteur ne peut ou ne veut pas identifier de façon plus précise. [...] [il] rejet[te] [le] référent dans l'anonymat » (2009: 364).

6 Les conventions de transcription sont disponibles en fin d'article.

7 Il s'agit donc d'un cas parmi quatre possibilités logiques qu'il est possible de distribuer en deux ensembles :

8 Cette situation de réciprocité des regards est d'autant plus probable qu'il semble que le récepteur d'un long tour de parole multi-unités ait tendance à regarder le locuteur précisément à la fin de la première unité syntaxique et prosodique de construction du tour (Rossano 2013), risquant dès lors de « rencontrer » le regard du locuteur.

\section{Pour citer cet article}

\section{Référence électronique}

Jérôme Jacquin, « S'opposer à autrui en situation de co-présence : la multimodalité de la désignation contre-argumentative », Semen [En ligne], 39 | 2015, mis en ligne le 19 novembre 2015, consulté le 28 janvier 2016. URL : http://semen.revues.org/10467

\section{À propos de l'auteur}

Jérôme Jacquin

Université de Lausanne (Suisse) / Victoria University of Wellington (Nouvelle-Zélande)

\section{Droits d'auteur}

(C) Presses universitaires de Franche-Comté 
